\title{
Modelagem paramétrica e prototipagem rápida no ensino de Expressão Gráfica
}

\author{
Parametric modeling and rapid prototyping \\ in teaching Graphic Expression
}

\section{Elton Cristovão da Silva Lima}

Arquiteto e Urbanista, Discente do Programa de PósGraduação em Urbanismo, Universidade Federal do Rio de Janeiro, Rio de Janeiro, Brasil

elton.lima@fau.ufrj.br ORCID:

https://orcid.org/00000002-8076-7150

\section{Leticia Teixeira Mendes}

Doutora em Arquitetura, Tecnologia e Cidade Docente do Departamento de Expressão Gráfica e do Programa de Pós-Graduação em Desenvolvimento Urbano, Universidade Federal de Pernambuco leticia.mendes@ufpe.br ORCID:

https://orcid.org/00000002-1011-1420

\section{Cristiana Maria Sobral Griz}

Doutora em Desenvolvimento Urbano

Docente do Departamento de Expressão Gráfica na Universidade Federal de Pernambuco cristiana.sgriz@ufpe.br ORCID:

https://orcid.org/00000002-5439-5680

\begin{abstract}
RESUMO
A inserção de novas tecnologias em várias áreas de atuação tem sido cada vez mais frequente e acelerada. Com o intuito de acompanhar essa crescente incorporação na rotina profissional do arquiteto, vê-se a necessidade da inclusão, também, no processo didático ao longo da formação profissional. 0 desenho é um meio de comunicação e pode ser feito de forma tradicional ou digital. Para isso, quanto maior o conhecimento e aplicação das propriedades e maneiras de representar formas geométricas, maior é a liberdade criativa do indivíduo. Este trabalho propõe a introdução do conceito de um sistema generativo de projeto (Gramática da Forma), aliado a modelagem paramétrica e a prototipagem rápida para dar suporte ao ensino de temas de geometria gráfica. A inserção da parametrização foi feita em um experimento, utilizando o software Rhinoceros e o plug-in Grasshopper, que vinculou o conteúdo de polígonos à concepção de cobogós por meio de uma gramática da forma paramétrica. 0 experimento mostrou que a modelagem paramétrica, ainda que se utilize das tecnologias computacionais, requer conhecimentos de geometria gráfica para explorar as potencialidades de maneira consciente e eficiente.
\end{abstract}

\section{Palavras-chave: Gramática da Forma; Design paramétrico; Geometria Gráfica; Método de ensino.}

\begin{abstract}
The insertion of new technologies in different fields has been increasingly frequent and accelerated. Therefore, in order to keep track of this growing incorporation of technologies into the professional routine of the architect, it is seen the need for inclusion also in the didactic process throughout professional qualification. Drawing is a means of communication and may be done in a traditional or digital way; and for that, the greater the knowledge and application of the properties and ways of representing geometric shapes, the greater the individual's creative freedom. This work proposes the introduction of the concept of a generative design system (Shape Grammar), combined with parametric modeling and rapid prototyping to support the teaching of graphic geometry themes. The parametric insertion was done during an experiment, using the Rhinoceros software and the Grasshopper plug-in, which linked the content of polygons for designing cobogos by means of a parametric shape grammar.-The experiment showed that parametric modeling, although using computational technologies, requires knowledge of geometry to explore potentialities in conscious and efficient way.
\end{abstract}

Keywords: Shape Grammar; Parametric design; Graphic Geometry; Teaching method. 


\section{INTRODUÇÃO}

A introdução de novas tecnologias nas áreas de projeto se deve, principalmente, à rápida evolução da indústria computacional. Esse novo paradigma tem gerado ferramentas e possibilidades eficientes na rotina de vários profissionais, como artistas, arquitetos, designers e engenheiros. Recentemente, a partir da inclusão das ferramentas de prototipagem rápida (PR) e fabricação digital (FD), tornou-se viável o uso de modelos geométricos digitais diretamente na produção de artefatos e componentes em escala real.

A prototipagem rápida é a materialização de modelos físicos para testes - maquetes e protótipos em escala reduzida ou em 1:1 - como uma das etapas do processo projetual. (Mitchell \& McCullough, 1995). Incluem técnicas como corte a laser, corte em vinil e fresas. Segundo Kolarevic (2003), a prototipagem rápida está inserida dentro da área abrangida pela fabricação digital. Esta, por sua vez, materializa o produto final ou parte dele, incluindo determinadas técnicas para construção de edifícios ou partes dele, fôrmas e peças finais com equipamentos CNC (Pupo, 2009).

Concomitantemente aos avanços tecnológicos na área de projeto, os docentes de disciplinas de projeto e representação gráfica têm se adaptado e proposto novos métodos de ensino, incorporando essas inovações no processo didático. Na área do ensino de expressão gráfica, essa mudança que vem acontecendo há algumas décadas (Pupo, 2008; Pupo et al., 2008) e está sendo acelerada com a intensão de que os jovens profissionais não fiquem descompassados em comparação com o mercado de trabalho. Segundo Oxman (2008, p. 99, tradução nossa):

A busca por novas estruturas educacionais se deve aos impactos pedagogicamente únicos do projeto digital. Vários pesquisadores e educadores começaram a abordar a necessidade de integrar o projeto digital na educação de projeto arquitetônico, investigando várias formas de agenda pedagógica. ${ }^{1}$

0 estudo das técnicas de geometria gráfica se integra à área de comunicação. Assim como a linguagem falada e escrita possui uma gramática, a linguagem gráfica também possui para seja bem compreendida, tanto por quem escreve, quanto por quem lê. Enquanto as duas primeiras formas de linguagem, a expressão é por meio de palavras, a gráfica é expressa, tradicionalmente, através de desenhos.

Contudo, como comentado, ferramentas tecnológicas, como a modelagem paramétrica, têm, aos poucos, substituído o desenho tradicional mesmo os que são feitos em "pranchetas digitais"2, através de "modelagem virtual” de formas geométricas. É importante salientar que, assim como o

${ }^{1}$ No original: The Search for new educational frameworks is due to the pedagogically unique impacts of digital design. Various researchers and educators have begun to address the need to integrate digital design in architectural design education investigating various forms of pedagogical agenda.

2 o software AutoCAD, por exemplo, pode ser considerado uma "prancheta digital" porque os desenhos feitos nele possuem a mesma lógica dos desenhos que são feitos na prancheta tradicional com lápis e papel. 
desenho, para executar as modelagens virtuais continua sendo fundamental o conhecimento da geometria gráfica.

Isso porque, como argumenta Florio (2011), aqueles que detém o conhecimento dos métodos de geometria gráfica possuem maior autonomia criativa. Isto é, quanto maior a compreensão das propriedades das formas geométricas e das suas possibilidades de representação, maior a competência e liberdade criativas. Assim, a geometria gráfica pode ser classificada como a ciência fundamental para a elaboração do projeto e para a pesquisa de forma.

Diante do exposto, o Departamento de Expressão Gráfica da Universidade Federal de Pernambuco, encarregado das disciplinas que abordam competências da geometria gráfica e diferentes abordagens para representar graficamente um projeto, tem reformulado os conteúdos e a metodologia de ensino de algumas disciplinas. Uma delas é a de Desenho Geométrico, responsável pelo conhecimento das propriedades gráficas de todas as formas geométricas bidimensionais e sua aplicação na arquitetura, urbanismo e paisagismo.

Visando a introdução não somente do conhecimento de novas tecnologias de concepção e representação de formas geométricas, mas também de uma nova metodologia projetual, foi incorporado um sistema generativo de projeto (Gramática da Forma, do inglês Shape Grammar) como apoio ao ensino do conteúdo do tema polígonos. De acordo com Fischer e Herr (2001), um sistema generativo é uma metodologia sistemática para produção de soluções projetuais, não apenas para gerar produtos, mas também processos.

Este trabalho apresenta uma proposta de renovação do ensino de geometria gráfica baseado na Gramática da Forma (Stiny e Gips, 1971), implementado através de ferramentas digitais, especialmente, o uso da modelagem paramétrica (Monedero, 2000) e prototipagem rápida. 0 objetivo é contribuir para inclusão de novos métodos de ensino de projeto, assim como no desenvolvimento de novas ferramentas e abordagens projetuais introduzidos na formação dos alunos.

Com o intuito de rever o ensino da geometria por meio da introdução de diferentes abordagens projetuais, este artigo apresenta o experimento realizado em uma disciplina de geometria gráfica para o curso de Arquitetura e Urbanismo, utilizando o projeto paramétrico (parametric design) e o formalismo da Gramática da Forma. Pretende-se analisar se a introdução dessas teorias pode auxiliar no entendimento mais interativo dos elementos geométricos e suas propriedades. Dessa forma, a parametrização e o projeto baseado em regras (rule-based design) foram utilizados visando otimizar o processo projetual e a geração de alternativas. 


\section{FUNDAMENTAÇÃO}

A temática do método em arquitetura tornou-se mais discutida na segunda metade do século XX, na ocasião em que arquitetos e engenheiros atentos ao panorama científico buscavam esclarecer o processo projetual visando inserir o computador de modo mais eficiente tanto na prática de projeto quanto no processo de ensino e aprendizagem. Surge assim o Movimento dos Métodos de Projeto (do inglês Design Methods Movement) consistiu em um período de conferências e publicações acerca dos métodos projetuais nas áreas de arquitetura, engenharia e desenho industrial na década de 1960, tendo significativa influência sobre o ensino e a prática dessas áreas de conhecimento (Mendes, 2014).

Nesse contexto de discussões sobre métodos de projeto, uma das abordagens foi a parametrização. Esta estratégia consiste em definir as relações topológicas, já que a definição de medidas precisas ocorre numa fase posterior do projeto. Geralmente essas dimensões podem ser selecionadas a partir de um intervalo desejado, com valores mínimos e máximos, resultando em uma maior variedade de soluções (Monedero, 2000).

De acordo com Florio (2011, p.44), para compreender e projetar formas de grande complexidade, os conhecimentos de geometria são essenciais. Assim, o autor sugere que se "faça uma re-valorização destes conhecimentos diante das facilidades trazidas pelas novas tecnologias de representação e de simulação de espaços".

Nesse contexto, os ambientes de modelagem paramétrica, como o Grasshopper ${ }^{3}$, por exemplo, ainda se constituem como ferramentas pouco exploradas no ensino de geometria. Os usuários destes softwares conseguem desenvolver modelos através de um processo indireto de geração de formas, sendo imprescindíveis os conhecimentos de geometria gráfica, programação computacional, matemática e lógica para otimizar o uso dessas ferramentas no processo projetual (Pereira e Vaz, 2013).

Segundo Oxman (2006), as técnicas de modelagem paramétrica são bases para o modelo de design associativo de formação ${ }^{4}$, viabilizando a exploração da geometria associativa, através da qual as relações entre os objetos paramétricos são explicitamente descritas, constituindo interdependências entre os vários objetos. Valores diferentes atribuídos a parâmetros de uma estrutura geométrica podem criar inúmeras variações, enquanto as condições topológicas ${ }^{5}$ são mantidas. Assim, pode-se afirmar que

\footnotetext{
3 É um plug-in que usa o software Rhinoceros como visualizador da geometria implementada no seu ambiente de programação visual. 0 Grasshopper utiliza componentes para montar qualquer combinação de parâmetros com a finalidade de obter um resultado geométrico no Rhinoceros. (Vaz, 2011).

4 Segundo a revista israelense Architecture of Israel Quarterly, o design associativo baseia-se em parâmetros variáveis e restrições. Ao passo que os parâmetros definem os valores dos elementos, as restrições determinam as relações entre eles, prescrevendo seu comportamento.

5 Segundo Kolarevic (2003), topologia refere-se a relações e interconexões dentro de um contexto espacial, e não sobre formas específicas. Uma construção topológica seria representada de diversas maneiras. Assim, topologia é mais sobre relação espacial do que distinção espacial.
} 
a modelagem paramétrica necessita de um ambiente computacional e usa algoritmos $^{6}$ para estabelecer definições, além de buscar a otimização de soluções.

Concomitantemente, desde 1960, várias abordagens estruturalistas ${ }^{7}$ vêm sendo empregadas no processo de projeto em arquitetura. Uma delas é o projeto baseado em regras - rule-based design (Broadbent, 1970), que consiste em definir situações em que determinado elemento pode ser conectado a outro sendo pré-definida a maneira como isso pode acontecer. Esse método permite a geração de inúmeros resultados diferentes, tendo em vista que a ordem de aplicação das regras influencia nas composições. Dentro desse contexto, o formalismo da Gramática da Forma permite a combinação dessas duas abordagens de projeto: a parametrização e o uso de regras compositivas (rule-based design) originando um número ainda maior na geração de possíveis soluções (Mendes, 2018).

A Gramática da Forma é um sistema algorítmico criado na década de 1970 por George Stiny e James Gips. Constitui-se de um sistema de geração de formas baseado em regras e foi desenvolvido a partir das teorias da gramática generativa do linguista Noam Chomsky e do sistema de produção do matemático Emil Post (Celani et al., 2006). No decorrer dos anos, a Gramática da Forma tem sido aplicada para resolver diversos problemas de projeto, buscando gerar soluções a partir de uma forma inicial e da aplicação sucessiva de regras de forma (Duarte, 2007; Knight, 2000; Celani et al., 2006).

Uma gramática da forma é composta por uma forma inicial, um conjunto finito de formas primitivas que compõe um vocabulário, e um conjunto finito de regras de transformação do tipo $\mathrm{A} \rightarrow \mathrm{B}$ (antes e depois). As regras podem ser, dentre vários exemplos, de adição, subtração, subdivisão, movimentação e substituição (Stiny, 1980), e por meio delas ocorrem as transformações geométricas que são aplicadas no vocabulário de formas. Isto é, a regra contém uma forma que deverá ser identificada (forma do lado esquerdo) e uma relação espacial do lado direito da regra (que substituirá a forma da esquerda). Com as regras estabelecidas, é possível aplicá-las sistematicamente e gerar as derivações como visto na figura 1 a seguir.

\footnotetext{
${ }^{6}$ De modo simplificado, pode-se comparar um algoritmo à uma receita. Técnica ou método formado por um conjunto finito de regras ou operações precisas, evidentes e simples que ao serem seguidas resultam na realização de uma ação. (Martino, 2015).

7 Consoante Colin (2009), o estruturalismo é abordado pelas ciências humanas com o objetivo de explorar um campo específico como um sistema complexo de partes que se interrelacionam. De acordo com Assiter (1984), nesta linha de pensamento sobre o estruturalismo existem quatro ideias fundamentais: a estrutura determina a posição de cada elemento como um todo; todo sistema possui uma estrutura; existem leis estruturais; as estruturas sustentam a aparência do significado.
} 
Figura 1: Gramática da forma para geração dos Jardins Mughul - exemplo de forma inicial, regra de subdivisão e derivação.

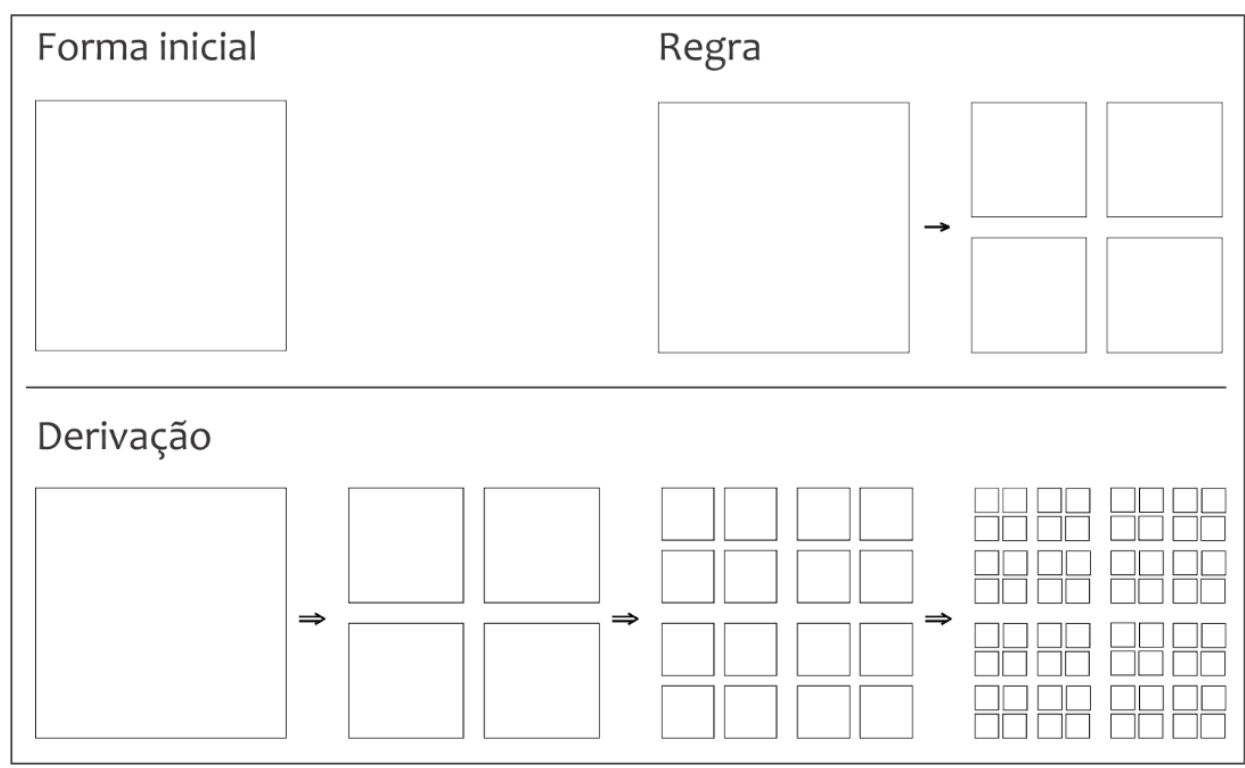

Fonte: os autores (2020), baseado em Stiny e Mitchell (1980).

A partir de então, vários tipos de gramática foram criados e aplicados na arquitetura como é o caso da gramática da forma geradora de vilas no estilo Paladiano a partir do estudo das vilas de Palladio desenvolvido por Stiny e Mitchell em 1978. Bem como a gramática de personalização de habitação social proposta por José Pinto Duarte baseada nas habitações de Álvaro Siza, na Malagueira em 2007. (Mendes, 2010.)

Segundo Duarte (2007), as gramáticas da forma podem ser originais ou analíticas. No primeiro caso, elas são desenvolvidas para gerar novos projetos que atendam exigências pré-estabelecidas. Já as analíticas são usadas como ferramenta de análise de um corpus - conjunto de projetos ou formas pré-existente, que são descritos através de uma linguagem única que os represente.

Pode-se incluir ainda uma terceira classificação das gramáticas, a paramétrica. Estas são implementadas em ambiente computacional com parâmetros manipuláveis. Segundo Celani et al. (2006), as primeiras gramáticas já possibilitavam deixar determinados valores (parâmetros) em aberto a serem estabelecidos no momento da implementação, permitindo a criação de uma gramática da forma paramétrica apta a produzir uma grande quantidade de resultados. De acordo com Santos (2009), uma gramática pode ser considerada como sistema generativo paramétrico quando a aplicação de determinada regra afeta um conjunto de sub-regras e a partir do momento que um número finito de entrada de dados, pode-se gerar uma quantidade indefinida de soluções.

Com a evolução das tecnologias computacionais, a implementação de Gramáticas da Forma em ambiente de modelagem paramétrica tornou-se possível e mais recorrente em diversas áreas como design, artes e arquitetura. 


\title{
3. MÉTODO
}

0 método proposto para o exercício projetual é baseado na metodologia da pesquisa-ação, proposta por Kurt Lewin, na década de 1940 e na prática reflexiva, proposta por Schön, em 1983 (Lewin, 1946; Schön, 1983). A metodologia desenvolvida por Lewin é essencialmente uma estratégia para o desenvolvimento de professores e pesquisadores que fazem uso de suas pesquisas para aprimorar sua didática e consequentemente, ajudar no aprendizado de seus alunos.

Em seu livro intitulado "Educando o profissional reflexivo: um novo design para o ensino e a aprendizagem", Schön (2000) além de apresentar a experimentação dos ateliês de projeto como um modelo educacional para a "reflexão-na-ação" 8 , também alega que não existe uma ciência que ensine a prática de projeto, além dos próprios ateliês como sendo um exemplo típico de ensino prático reflexivo. Além disso, sobre o processo de prática de projeto nos ateliês de arquitetura, ele pontua:

\begin{abstract}
Implicam um tipo de aprender fazendo, em que os alunos começam a praticar, juntamente com os que estão em idêntica situação, mesmo antes de compreenderem racionalmente o que estão a fazer. Nos ateliês de projeto arquitetônico, por exemplo, os alunos começam por desenhar antes de saberem o que é o projeto. Nos primeiros tempos toda a gente se queixa da confusão. (SCHÖN, 1997, p.89).
\end{abstract}

Deste modo, a experiência apresentada nesta pesquisa - um workshop de modelagem paramétrica para o desenvolvimento de cobogós ${ }^{9}$ - demonstra a aplicação da metodologia com o intuito de atualizar o conteúdo de desenho geométrico através de um experimento que utiliza a parametrização e a prototipagem rápida no ensino da graduação em arquitetura e urbanismo.

Como observado anteriormente, o formalismo da Gramática da Forma já foi utilizado em diversas áreas do conhecimento para análise e geração desde cadeiras (Knight, 1980; Barros et al., 2011) e cerâmicas (Costa e Duarte, 2015) até habitações (Duarte, 2007) e planos urbanísticos (Beirão, 2012; Duarte, 2012). Neste trabalho, o elemento arquitetônico definido como cobogó foi adotado como objeto de estudo por se tratar de uma composição formal e passível de aplicação do conteúdo da disciplina (polígonos e suas propriedades formais). Assim, foi sugerido que os alunos se concentrassem

\footnotetext{
8 Segundo Micheletto (2008), a reflexão-na-ação carrega um saber que pode ser entendido como conhecimento técnico ou solução de problemas, norteia toda atividade humana e se expressa no saberfazer.

9 Conforme Santana Neto e Silva (2016), o cobogó funciona como uma estratégia passiva para amenização climática bastante difundida na arquitetura moderna brasileira. Constitui-se como um elemento vazado utilizado como sistema de vedação e proteção com a intenção de permitir a ventilação e ao mesmo tempo filtrar a incidência solar.
} 
somente na composição estética e formal do cobogó, analisando basicamente a relação entre as formas bidimensionais e sua composição.

A gramática apresentada aos alunos era formada por 3 elementos geométricos básicos - círculo, triângulo e quadrilátero - como vocabulário e relações espaciais que relacionavam estes elementos entre si além de incluir o diagrama Voronoi ${ }^{10}$ - tipo de decomposição espacial através da inserção de pontos aleatórios em determinado plano. As regras consistiam na adição de um elemento dentro de outro que compunha o vocabulário. Nesse sentido, era possível, a partir de parâmetros, modificar os tamanhos e os pontos de inserção. Por fim, na derivação os alunos faziam a composição de uma parede de cobogós onde havia possibilidade de alterar a quantidade de peças no eixo x e y.

Nesse contexto, foi usado o ambiente de modelagem paramétrica visual Grasshopper associado ao software de modelagem 3D Rhinoceros que proporcionaram a inserção e manipulação dos parâmetros. Essa ferramenta de implementação de algoritmos generativos é comumente usada em pesquisas embasadas no uso de gramáticas da forma e modelagem paramétrica em workshops para alunos de graduação (Beirão et al., 2015, Vaz, 2011).

A linguagem de programação visual viabiliza a construção de geometrias por meio da conexão de componentes e parâmetros (Khabazi, 2010). A seguir, na Figura 2, é possível, a partir da construção de um cilindro, identificar alguns destes elementos; um exemplo de componente (extrude), barra de rolagem (slider) destacada em verde, dados de entrada e saída (input e output) que desempenham funções predefinidas, permitindo criar, desconstruir e alterar formas. Quanto às interfaces, à direita tem-se a visualização, através do software de modelagem 3D Rhinoceros, do cilindro implementado no ambiente de programação visual Grasshopper.

Figura 2: interface do ambiente de modelagem paramétrica visual Grasshopper.

10 Diagrama Voronoi é um modelo de análise que possibilita o estudo das regiões de influência de pontos de interesse determinadas por suas localizações em relação ao conjunto de dados. (Moura, 2009). 


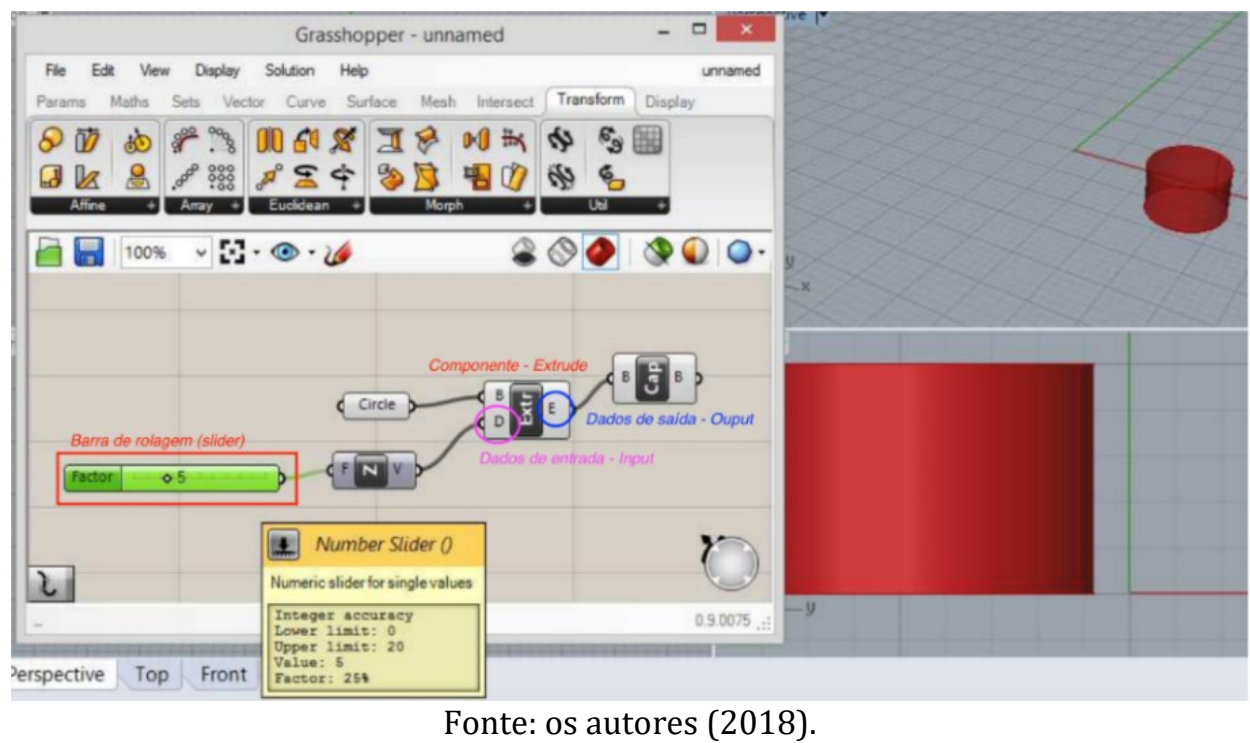

O código utilizado na gramática de cobogós, por sua vez, também faz uso desses elementos de entrada e saída de dados, através dos quais, é possível fazer modificações nas peças em comprimento, largura, espessura, ponto de inserção das formas, aplicação das regras, adição de cor e recursão na derivação. (Figura 3).

A figura 3, a seguir, expõe o código dividido em partes para melhor compreensão do processo da lógica de implementação. A primeira parte (a) se refere à construção do vocabulário de formas; seguindo para as regras (b); as regras passíveis de modificações que foram usadas na derivação (c), neste caso os sliders em verde permitem modificar formas, escala e relações espaciais. Em amarelo, a parte do código que permite definir qual a forma da peça do cobogó para que seja feita a derivação (d), bem como a quantidade de peças nos eixos $\mathrm{x}$ e $\mathrm{y}$. Em (e), tem-se o componente que permite modificar as cores na visualização das peças. 
Figura 3: fluxo do código implementado para o experimento.

a) Vocabulário de formas

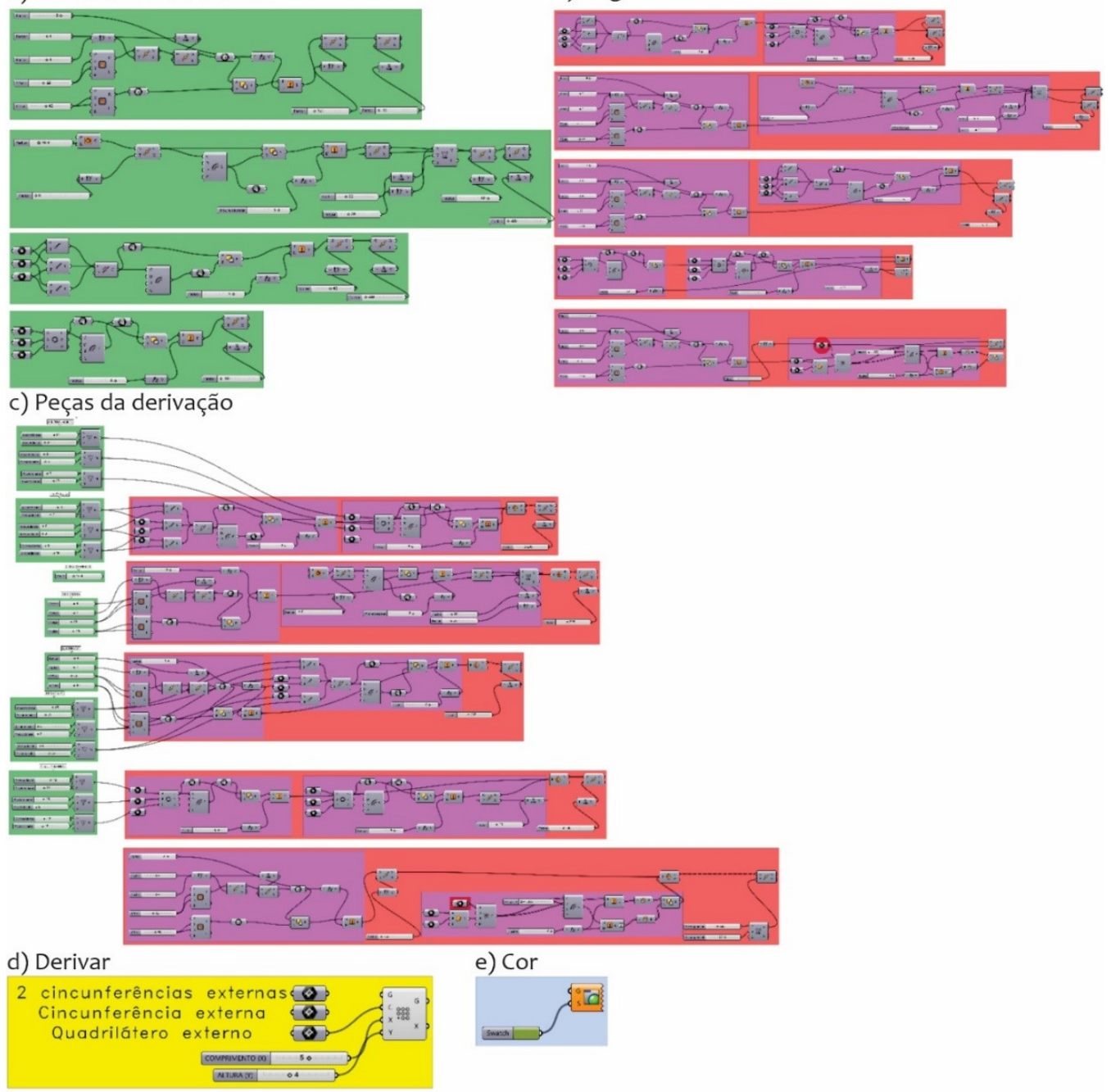

Fonte: os autores (2018).

\section{O EXPERIMENTO: UMA GRAMÁTICA DA FORMA PARA GERAÇÃO DE}

\section{coBoGós.}

Como já mencionado, a disciplina de Geometria Gráfica (Desenho Geométrico) é responsável pelo ensino das propriedades e relações das formas geométricas bidimensionais. A experiência, a princípio, foi realizada com o conteúdo de 'polígonos', visando o aprendizado das propriedades formais e os elementos que os constituem. Esses conhecimentos permitem que os alunos construam vários tipos de polígonos, tanto da maneira tradicional, com lápis e papel, quanto de maneira digital, por meio de um ambiente de modelagem paramétrica.

O workshop foi ministrado para 42 estudantes matriculados no curso de arquitetura. As atividades foram realizadas em duplas ou grupos de três 
pessoas. Primeiramente, os/as estudantes foram orientados a criar três elementos geométricos básicos - círculo, quadrilátero e triângulo - usando ferramentas disponíveis no Grasshopper. Para esta atividade foi imprescindível o conhecimento prévio dos conceitos de desenho geométrico para construção dessas figuras, como raio, diâmetro, incentro, ortocentro, circuncentro de uma circunferência, além dos tipos de triângulos, quadriláteros e polígonos com cinco ou mais lados etc. A Figura 4, a seguir, expõe três exemplos de implementações: (A) construção de circunferências e arcos de concordância; (B) exemplo de quadrilátero; (C) exemplo de triângulo.

Figura 4: exemplo de construção de formas geométricas utlizando componentes internos do Grasshopper. Forma visível à esquerda e código implementado à direita.

A)
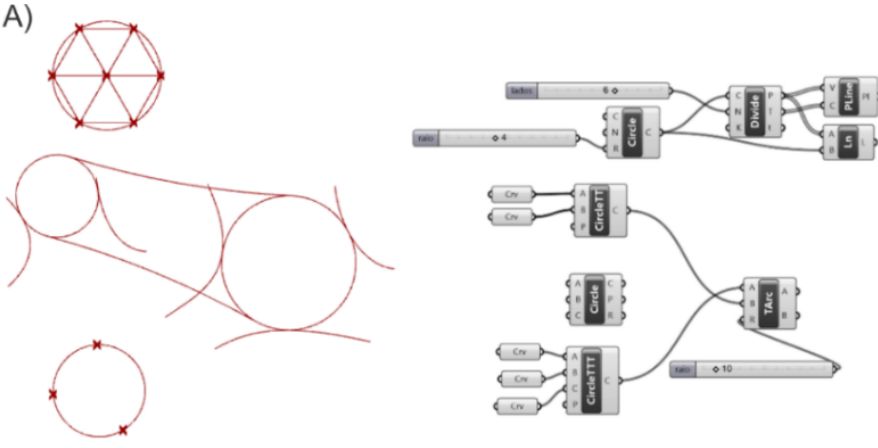

B)

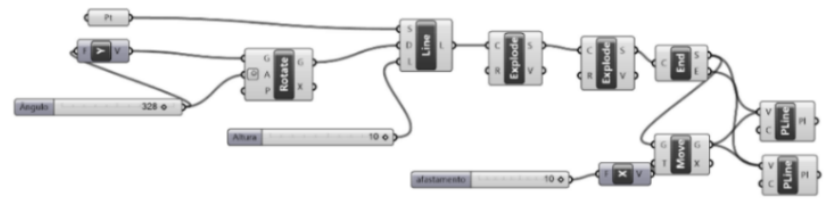

C)

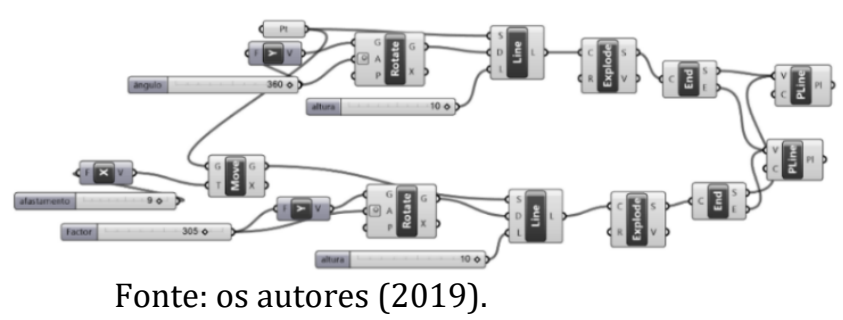

Logo após os/as alunos(as) se familiarizarem com a interface do Grasshopper, eles testaram a gramática da forma cedida que foi previamente implementada. Para isso, os/as participantes manipularam os dados de entrada nos códigos para alterar os parâmetros conforme exemplifica a Figura 5. As regras implementadas possibilitavam modificar a quantidade de lados do polígono e aplicar transformações geométricas, como rotação e translação. Isso permitiu gerar inúmeros modelos de cobogós a partir de polígonos como forma inicial e da modificação de suas formas e relações espaciais.

Figura 5: implementação do código responsável pelo vocabulário de formas da gramática de cobogós e suas respectivas formas (A) e exemplos de cobogós criados pelos alunos a partir da implementação (B). 


\section{A)}
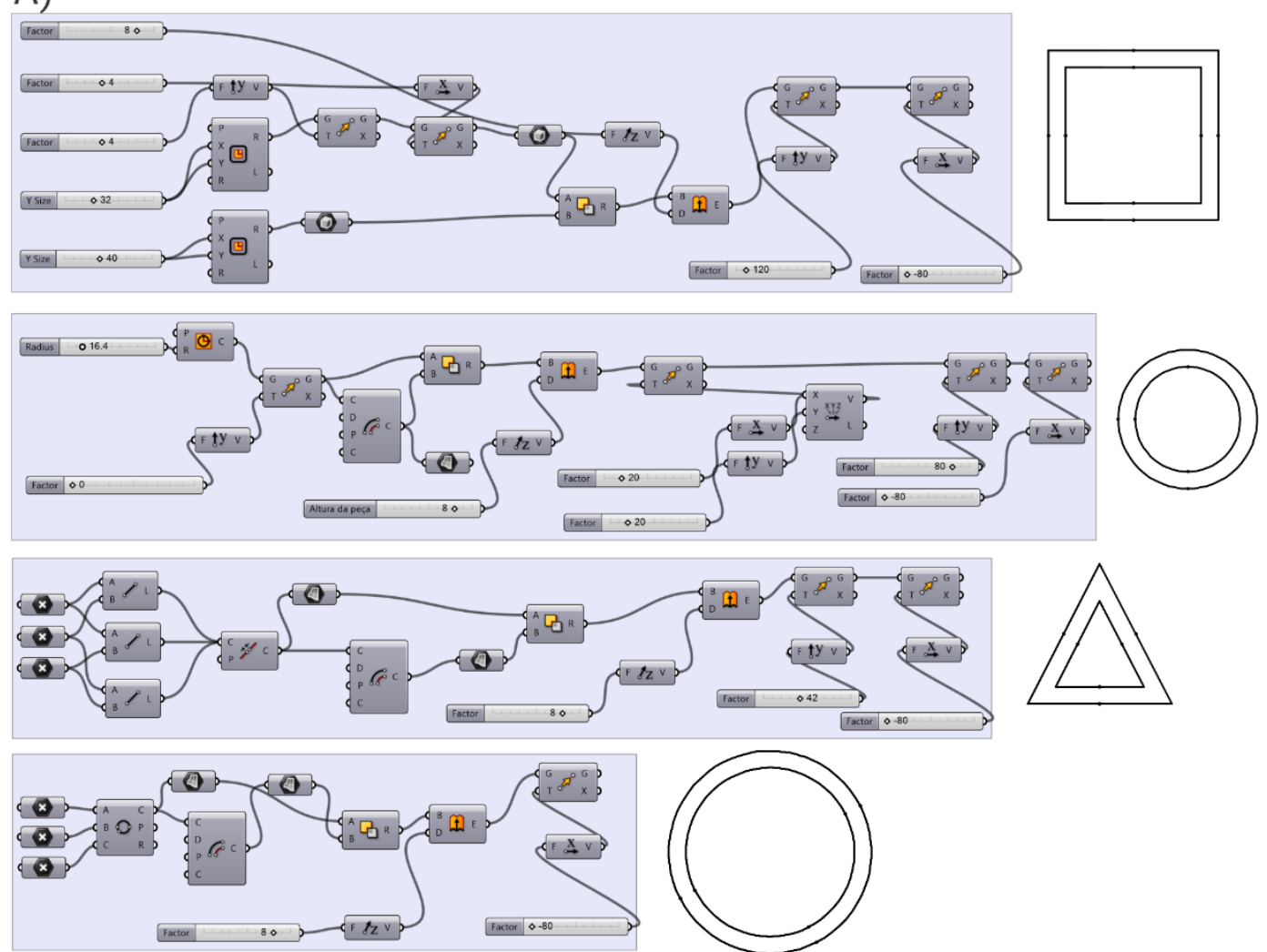

B)
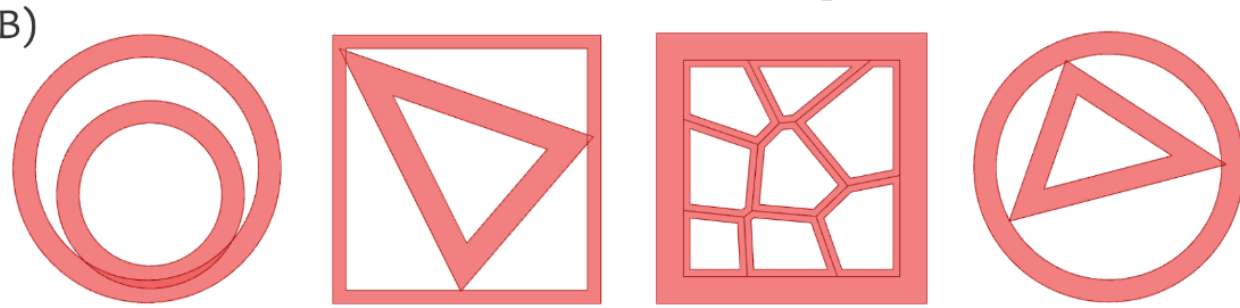

Fonte: os autores (2019).

Posteriormente à criação dos cobogós, estes foram produzidos com impressoras 3D como parte dos resultados do experimento. Para tanto, foi usada a tecnologia aditiva de prototipagem rápida conhecida como Modelagem por Decomposição Fundida (Fused Deposition Modeling - FDM). Conforme Pallasma (2011), a materialização é importante por contribuir no desenvolvimento do pensamento de projeto, permitindo a comunicação tanto através do tato quanto da visão (Figura 6). 
Figura 6: (A) processo de impressão 3D; (B) exemplo de um cobogó desenvolvido pelos alunos; (C) protótipos de cobogós impressos.

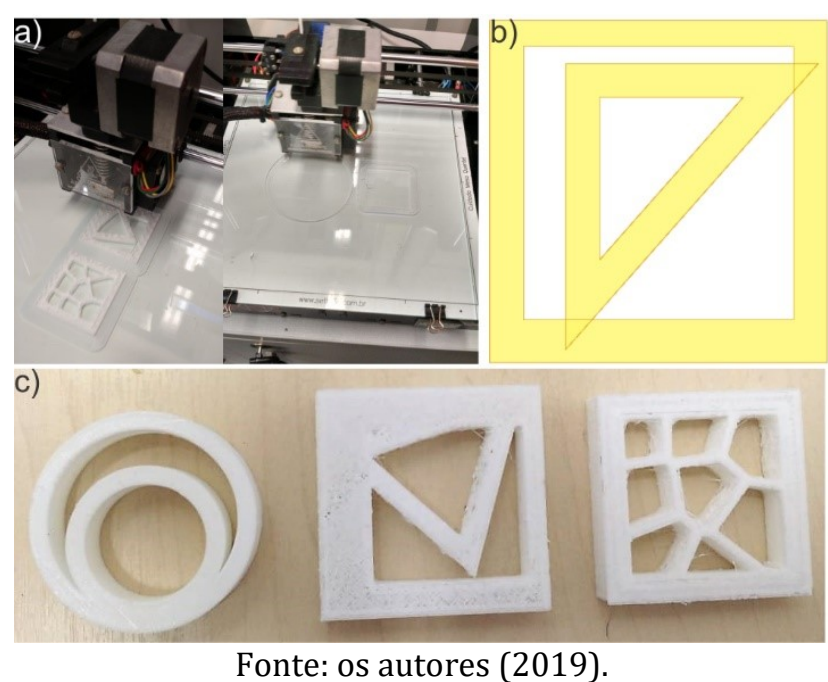

A técnica de prototipagem tornou possível materializar diversos cobogós gerados pela mesma gramática da forma, possuindo assim os mesmos princípios compositivos, contudo, com inúmeras variações formais (Figura 7).

Figura 7: exemplo de composição de um painel com diferentes cobogós desenvolvidos pelos alunos a partir da gramática da forma paramétrica.

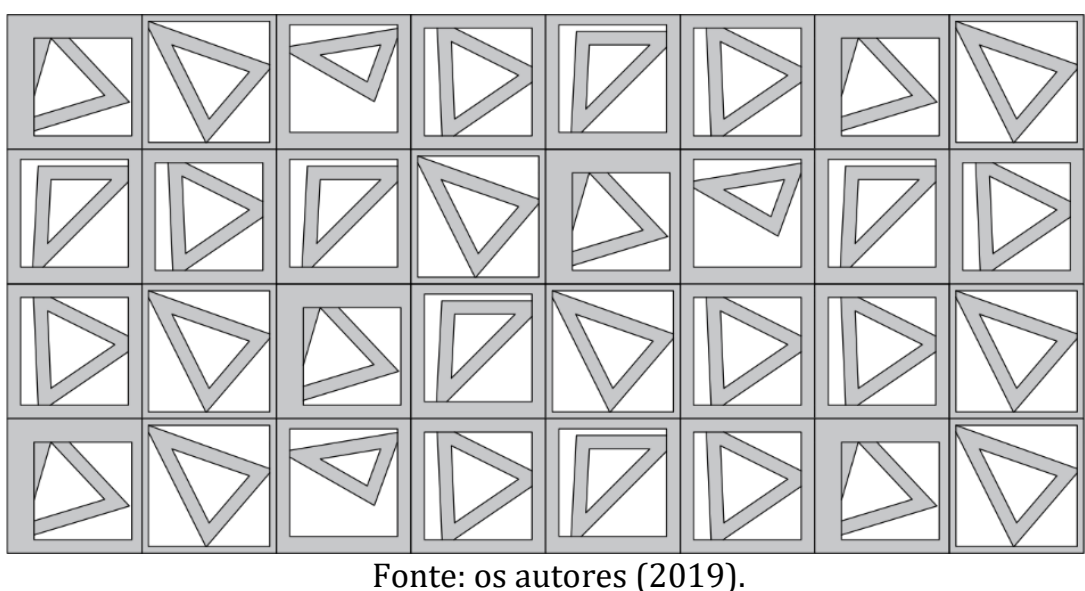

A Gramática da Forma associada ao potencial da prototipagem rápida provocou questionamentos contrapondo os modelos de produção em série (mass production) e o conceito de personalização em massa (mass customization) estabelecido por autores como Pine (1993), Stan Davis (1996), Tseng e Jiao (2001), e Kaplan e Haenlein (2006). Nesse contexto, a Gramática da Forma possibilita gerar inúmeras alternativas (ou vários cobogós) de forma dinâmica e automatizada, enquanto a prototipagem rápida permite sua materialização individualizada, isento de custos extras. 


\section{RESULTADOS E DISCUSSÃO}

O presente experimento parte da discussão acerca da introdução de diferentes abordagens projetuais e das tecnologias digitais no ensino de Arquitetura, assim propôs o uso do formalismo da Gramática da Forma aliado ao projeto paramétrico para criação de cobogós como instrumento para abordar conteúdos de Geometria Gráfica, como a construção e propriedades dos polígonos. Além disso, demonstrou que é imprescindível os conhecimentos de geometria para explorar as potencialidades da ferramenta de modo consciente e eficiente. Isto favorece a dinâmica de transitar entre procedimentos dedutivos referentes às construções gráficas e indutivos no que se refere às associações (Terzidis, 2006).

Para analisar se o experimento foi eficiente para o ensino do conteúdo de polígonos, foi feito um questionário a ser respondido pelos participantes. Este continha perguntas relacionadas ao nível de complexidade da atividade, se o aluno tinha conhecimento prévio de programação e se a utilização do ambiente de modelagem paramétrica visual ajudou na compreensão dos conteúdos. Ainda foi questionado se o conhecimento prévio de geometria ajudou a entender a lógica do plug-in, se foi possível compreender as potencialidades do uso de um sistema generativo e por fim, se o aluno usaria as abordagens projetuais apresentadas no desenvolvimento de um projeto.

Dos 42 alunos participantes do experimento, apenas três já haviam trabalhado com programação utilizando a linguagem Java. Por causa desta experiência prévia, estes alunos avaliaram com o conceito "muito fácil" a interação com o ambiente de programação visual Grasshopper - diferente da relativa dificuldade relatada pelos demais alunos participantes (Figura 8).

Figura 8: gráfico do nível de dificuldade de interação com o Grasshopper, desenvolvido a partir de um questionário aplicado aos alunos participantes dos workshops. Muito fácil (3 alunos), fácil (7 alunos), moderado (16 alunos), complexo (14 alunos) e muito complexo (2 alunos).

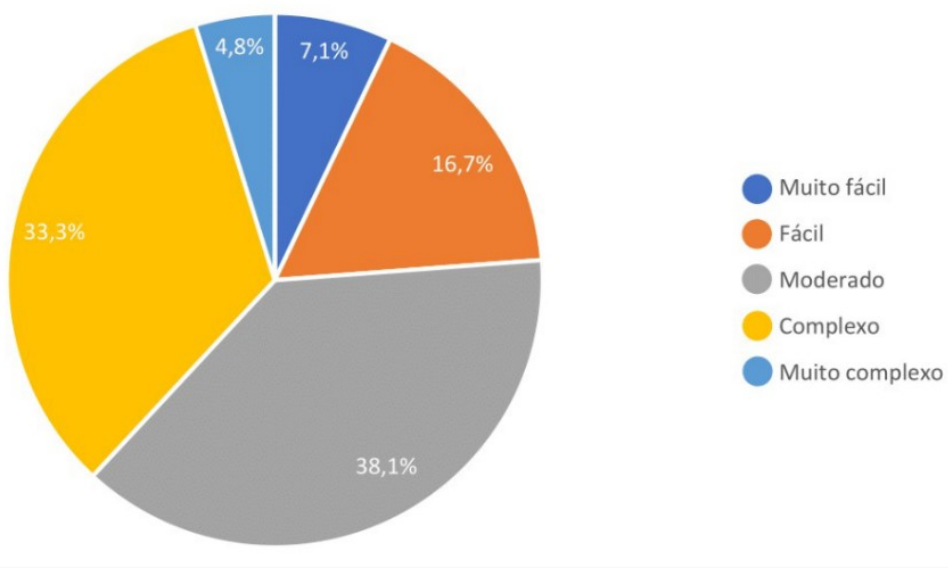

Fonte: os autores (2019).

Conforme a avaliação dos alunos, 38,1\% consideraram "moderada" sua interação com o Grasshopper. Isso pode ser justificado pelo fato de ter sido utilizado um ambiente de modelagem paramétrica visual, onde não era 
necessário conhecimento de programação com códigos escritos, e, ainda assim, os participantes puderam criar e manipular as definições por meio de componentes visuais como visto na figura 1. Além disso, alguns ícones podem ser encontrados intuitivamente e sua visualização é passível de ajustes, possibilitando a alteração entre o desenho gráfico representativo ou o próprio nome do componente (Figuras 4 e 5).

Quanto às atividades propostas, os alunos acharam mais fácil criar o código de programação para criação dos elementos geométricos paramétricos do que apenas a manipulação da gramática da forma que apresentava as definições prontas. Este resultado revela que a implementação da programação desenvolvida pelos alunos do início ao fim permite a compreensão da lógica subjacente sendo construída. Ou seja, fornecer a definição já pronta para se chegar a um resultado final de cobogós dificultou o entendimento do processo como um todo, fazendo com que os alunos apenas manipulassem os parâmetros, na maioria dos casos, de forma inconsciente e automática.

Já em relação à Gramática da Forma, foi perceptível nas aulas expositivas e no primeiro contato da turma com este conteúdo, que os alunos tinham dificuldade para entender o funcionamento da lógica deste sistema generativo. Porém, quando a gramática foi abordada de forma paramétrica, 81\% dos alunos (34) disseram ter compreendido melhor.

Segundo as respostas dos questionários, 90,5\% dos participantes (38) afirmaram ter entendido as potencialidades da abordagem projetual do projeto paramétrico. No entanto, menos de $50 \%$ da turma disse que usaria o design paramétrico como método projetual. Um provável motivo do desinteresse em utilizar essa abordagem novamente em outras oportunidades, pode ser devido à complexidade do ambiente de modelagem paramétrico visual e poucas oportunidades para utilização de diferentes abordagens projetuais no curso de Arquitetura e Urbanismo da instituição.

Vale ressaltar que os participantes reiteraram que é primordial o conhecimento das abordagens de projeto apresentadas (parametrização e projeto baseado em regras) no workshop para que a atividade pudesse ser melhor desenvolvida. Ademais, disseram que o conhecimento prévio de desenho geométrico facilitou a compreensão da lógica da implementação paramétrica. 92,9\% dos alunos (39) disseram que a compreensão de geometria (construção de polígonos, suas propriedades formais e seus elementos constitutivos) foi indispensável e permitiu maior fluidez na assimilação de como desenvolverem a implementação dos objetos no Grasshopper.

\section{CONSIDERAÇÕES FINAIS}

O experimento foi guiado pela crítica ao ensino tradicional de disciplinas que envolvem os saberes da Geometria Gráfica e a necessidade de 
introduzir uma mudança de paradigma (imposta pelo avanço das tecnologias digitais) na prática projetual. Partiu-se do pressuposto de que esses conhecimentos são fundamentais para a compreensão e manipulação de formas de grande complexidade, usando ferramentas contemporâneas.

No entanto, devido ao caráter introdutório da modelagem paramétrica e da Gramática da Forma, foram realizados exercícios mais simplificados com os estudantes. Porém, é possível afirmar que o percurso teórico e metodológico empregado obteve êxito por permitir aos participantes das oficinas compreenderem as possibilidades e as potencialidades dessas ferramentas de projeto.

Em meio as considerações já apontadas na avaliação dos resultados, destaca-se a importância do experimento realizado visando a atualização do conteúdo ministrado em disciplinas de geometria, sobretudo no que tange à introdução de diferentes abordagens de projeto, como o design paramétrico e o projeto baseado em regras, por exemplo. Esta experiência comprovou a urgência de se propor novas metodologias no ensino da geometria gráfica que integrem as tecnologias digitais, fomentando uma associação do conhecimento tradicional com a apropriação consciente dos recursos tecnológicos atuais.

Mediante as respostas dos alunos observou-se a importância de oferecer ferramentas que lhes permitam explorar de modo sistemático e objetivo (possibilitado pelo uso da Gramática da Forma e da modelagem paramétrica), grandes quantidades de alternativas de resultados de maneira dinâmica e automatizada.

Dessa forma, com a finalidade de contribuir na inserção de novas metodologias projetuais no ensino de disciplinas do curso de Arquitetura e Urbanismo, pretende-se desdobrar o método aplicado neste experimento, para outros conteúdos de disciplinas de Geometria Gráfica. Assim, seria possível empregar recursos paramétricos para o desenvolvimento de escadas, rampas e coberturas proporcionando mais tempo de assimilação do conhecimento do tema estudado e das ferramentas digitais, além de aplicá-los em problemas projetuais de maior complexidade.

\section{REFERÊNCIAS BIBLIOGRÁFICAS}

BARROS, M.; DUARTE, J.; CHAPARRO, B. Thonet Chair Design Grammar: A Step towards the Mass Customization of Furniture. In: CAAD FUTURES, Proceedings: Designing Together. Liège, 2011. p. 181-200.

BEIRÃO, J. N. CityMaker: Designing Grammars for Urban Design. Architecture and the Built Environment, n. 5, 2012. Delft: BK Books.

BRAIDA, Frederico et al. (Orgs.). 101 conceitos de arquitetura e urbanismo na era digital. São Paulo: ProBooks, 2016. 
COSTA E. C.; DUARTE J. P. Generic Shape Grammars for Mass

Customization of Ceramic Tableware. In: GERO, J; HANNA, S. (EDS) DESIGN COMPUTING AND COGNITION. v.14, 2015.

CELANI, G.; CYPRIANO, D.; GODOY, G.; VAZ, C. E. A gramática da forma como metodologia de análise e síntese em arquitetura. Conexão - Comunicação e Cultura. v. 5, p. 180-197, 2006. Caxias do Sul.

COLIN, S. As estruturas. Revista AU. Editora PINI: n. 181, Abril 2009, p.81.

DAVID, Stan. Future Perfect. Harlow: Addison-Wesley Pub Co, 1996.

DUARTE, J. P. Personalizar a habitação em série: Uma Gramática Discursiva para as Casas da Malagueira do Siza. 2007. Lisboa: Ed. Fundação Calouste Gulbenkian.

DUARTE, J. P.; BEIRÃO, J. N. Towards a methodology for flexible urban design: designing with urban patterns and shape grammars. Environment and Planning B: planning and design, v. 38, n. 5, p. 879-902, 2012.

FISHER, T.; HERR, C. M. Teaching generative design. 2001. Proceedings of the 4th International Generative Art Conference. Milan: Ed. SODDU.

FLORIO, W. Modelagem Paramétrica, Criatividade e Projeto: duas experiências com estudantes de arquitetura. Gestão e Tecnologia de Projetos. São Carlos, v. 6, n. 2, p.43-66, 2011.

KAPLAN, A.; HAELEIN, M. Toward a parsimonious definition of traditional and electronic mass customization. In: Journal of product innovation management 23, vol. 2, 2006.

KHABAZI, Z. Generative Algorithms using Grasshopper. Morphogenesis education, 2010.

KNIGHT, T. Shape Grammars in education and practice: history and prospects, proceedings of International Journal of Design Computing, v. 2, 2000. Disponível em: <http://www.mit.edu/ tknight/IJDC/>. Acesso em: 23 jul. 2012.

KNIGHT, T. The generation of Hepplewhite-style chai-back designs. Environment and Planning B. v.7, p. 227-238, 1980.

KOLAREVIC, B. Architecture in the digital age: design and manufacturing. Oxon: Taylor \& Francis Group, 2003.

MENDES, L. T. Habitação coletiva contemporânea: inovações metodológicas e tecnologia da informação no processo de projeto. Campinas, 2010. Dissertação (mestrado) - Faculdade de Engenharia Civil, Arquitetura e Urbanismo. Universidade Estadual de Campinas - UNICAMP.

MENDES, L. T. Personalização de habitação de interesse social no Brasil: o caso da implantação urbana em conjuntos habitacionais. Campinas, 2014. Tese de Doutorado - Faculdade de Engenharia Civil, Arquitetura e Urbanismo. Universidade Estadual de Campinas - UNICAMP. 
MENDES, L. T. Gramática da forma: aplicações em habitação social, 2018. In: CELANI, G., SEDREZ, M. (Org.) Arquitetura Contemporânea e Automação: Prática e reflexão. ProBooks: São Paulo, pp. 41-54.

MARTINO, J. A. Algoritmos evolutivos como método para desenvolvimento de projetos de arquitetura. Campinas, 2015. Tese (Doutorado) - Faculdade de Engenharia Civil, Arquitetura e Urbanismo. Universidade Estadual de Campinas - UNICAMP, 2015.

MICHELETTO, Ingrid Barbara Pereira. Ação-Reflexão-Ação: processo de formação continuada. II SEPED Seminário de Pedagogia Infância: Múltiplos Olhares UENP, 2008.

MITCHELL, W. J.; McCULLOUGH M. Digital Design Media. New York: Van Nostrand. Reinhold, 1995.

MONEDERO, J. Parametric design - a review and some experiences. Automation in Construction, v. 9, n.4, p. 369-377, 2000.

MOURA, Ana Clara M.. Aplicação do modelo de Polígonos de Voronoi em estudos de áreas de influência de escolas públicas: estudo de caso em Ouro Preto/MG. Revista Brasileira de Estudos Regionais, v. 3, p. 1-25, 2009.

OXMAN, R. Theory and Design in the first digital age. Design Studies, v. 27, 2006. London: Elsevier.

OXMAN, R. Digital architecture as a challenge for design pedagogy: theory, knowledge, models and medium. Design Studies, v. 29, p. 99-120, 2008. London: Elsevier.

PAIO, A.; TURKIENICZ, B. A Grammar for Portuguese Historical Urban Design. FUTURE CITIES [28th eCAADe Conference Proceedings / ISBN 978-09541183-9-6] ETH Zurich (Switzerland) 15-18 September 2010, p.349-358.

\section{PALLASMA, J. As Mãos Inteligentes: A sabedoria Existencial e}

Corporalizada na Arquitetura. Porto Alegre: Bookman, 2011.

PEREIRA, N. S.; VAZ, C. V. Parametrismo e Ensino de Geometria - As Superfícies de Felix Candela. 2013. Disponível em: <http://wright.ava.ufsc.br/ grupohipermidia/graphica2013/trabalhos/PAR AMETRISMO\%20E\%20ENSINO\%20DE\%20GEOMETRIA\%20AS\%20SUPERFI CIES\%20DE\%20FELIX\%20CANDELA.pdf>. Acesso em: jul. 2018.

PINE, B. Mass Customization - The New Frontier in Business

Competition. Boston, Mass.: Harvard Business School Press, 1993.

PUPO, R. T. Ensino da prototipagem rápida e fabricação digital para arquitetura e construção no Brasil: definições e estado da arte. PARC Pesquisa em Arquitetura e Construção, Campinas, SP, v. 1, p.1-19, 2008. ISSN 1980-6809.

PUPO, R. T. Inserção da prototipagem e fabricação digitais no processo de projeto: um novo desafio para o ensino de arquitetura. Campinas, 
2009, 240p. Tese de doutorado - Faculdade de Engenharia Civil, Arquitetura e Urbanismo - Universidade Estadual de Campinas.

PUPO, R.; DUARTE, J.; CELANI, G. Introducing digital fabrication into the architectural curriculum: two similar experiences in different contexts. Congresso ECAADE. Antwerpen, set. 2008.

SANTANA NETO, E. J.; SILVA, R. C. Computação material: um estudo sobre a atualização geométrica de elementos vazados na arquitetura. In: SIGRADI - XX CONGRESO DE LA SOCIEDAD IBERO-AMERICANA DE GRÁFICA DIGITAL. Buenos Aires, p. 42-49, 2016. Disponível em: $<$ http://pdf.blucher.com.br.s3-sa-east1.amazonaws.com/designproceedings/sigradi2016/479.pdf>. Acesso em: ago. 2018.

SANTOS, L. F. B. S. Sistemas Generativos de Projecto: Integração de Ferramentas Digitais no Projecto de Arquitectura. 2009. Dissertação (Mestrado em Arquitetura) - Instituto Superior Técnico, Universidade Técnica de Lisboa. Lisboa.

SCHÖN, D. The reflective practitioner: how professionals think in action. Nova York: Basic Books, 1983.

SCHÖN, D. Formar professores como profissionais reflexivos. In: NÓVOA, A. (Coord.). Os Professores e a sua Formação. 3. ed. Lisboa: Publicações Dom Quixote. 1997.

SCHÖN, D. Educando o Profissional Reflexivo: um novo design para o ensino e a aprendizagem. Porto Alegre: Artmed. 2000.

STINY, G.; GIPS, J. Shape Grammars and the Generative Specification of Painting and Sculpture, in C V Freiman (ed) Proceedings of IFIP Congress 71 (Amsterdam: North-Holland), p. 1460- 1465, 1971. Republished in O R Petrocelli (ed), The Best Computer Papers of 1971 (Philadelphia: Auerbach) p. 125-13.

STINY, G. Introduction to shape grammars. Environmental and Planning B. v.7, p. 343-351, 1980.

STINY, G.; MITCHELL, W. J. The grammar of paradise: on the generation of Mughul gardens. Environmental and Planning B. v.7, p. 209-226, 1980.

TERZIDIS, K. Algorithmic Architecture. Burlington: Elsevier, 2006.

TSENG, M.; JIAO, J. Mass customization. In: Handbook of Industrial Engineering, Technology and Operation Management, 2001.

VAZ, C. E. V. Um método de ensino de projeto baseado em precedentes sistemas generativos e ontolologias aplicados no ensino de arquitetura paisagística. 2011. Tese de doutorado - Universidade Estadual de Campinas, Campinas. 